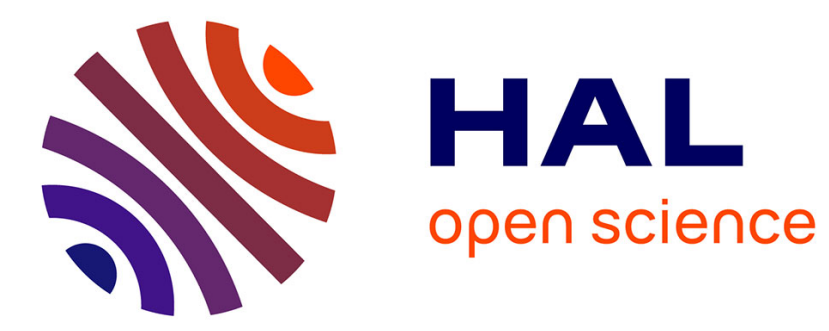

\title{
Thermalization of a two-phase fluid in low gravity: heat transferred from cold to hot
}

Régis Wunenburger, Yves Garrabos, Carole Lecoutre-Chabot, Daniel Beysens, John Hegseth

\section{> To cite this version:}

Régis Wunenburger, Yves Garrabos, Carole Lecoutre-Chabot, Daniel Beysens, John Hegseth. Thermalization of a two-phase fluid in low gravity: heat transferred from cold to hot. Physical Review Letters, 2000, 84 (18), pp.4100-4103. 10.1103/PhysRevLett.84.4100 . hal-03266082

\section{HAL Id: hal-03266082 https://hal.science/hal-03266082}

Submitted on 21 Jun 2021

HAL is a multi-disciplinary open access archive for the deposit and dissemination of scientific research documents, whether they are published or not. The documents may come from teaching and research institutions in France or abroad, or from public or private research centers.
L'archive ouverte pluridisciplinaire HAL, est destinée au dépôt et à la diffusion de documents scientifiques de niveau recherche, publiés ou non, émanant des établissements d'enseignement et de recherche français ou étrangers, des laboratoires publics ou privés. 


\title{
Thermalization of a Two-Phase Fluid in Low Gravity: Heat Transferred from Cold to Hot
}

\author{
R. Wunenburger, ${ }^{1}$ Y. Garrabos, ${ }^{1}$ C. Lecoutre-Chabot, ${ }^{1}$ D. Beysens, ${ }^{2}$ and J. Hegseth ${ }^{3}$ \\ ${ }^{1}$ Equipe du Supercritique pour l'Environnement, les Matériaux et l'Espace, Institut de Chimie de la Matière Condensée \\ de Bordeaux, Centre National de la Recherche Scientifique, Université Bordeaux I, F-33608 Pessac Cedex, France \\ ${ }^{2}$ Equipe du Supercritique pour l'Environnement, les Matériaux et l'Espace, Commissariat à l'Energie Atomique, \\ F-38054 Grenoble Cedex, France \\ ${ }^{3}$ Department of Physics, University of New Orleans, New Orleans, Louisiana 70148
}

(Received 3 December 1999)

\begin{abstract}
We present an experimental study of the thermal response to a positive temperature quench in twophase fluid $\mathrm{SF}_{6}$ in low gravity for temperature ranging from 10.1 to $0.1 \mathrm{~K}$ from the critical temperature. The temperature was measured simultaneously in the gas, the liquid, and the cell wall by thermistors and the density distribution was observed by interferometry. During the quench the gas temperature considerably exceeded the temperature of the heating walls (overheating up to 23\%). This striking observation is discussed in the light of the adiabatic heat transfer in this highly compressible fluid while the key role of the localization in low gravity of the gas and liquid phases is revealed.
\end{abstract}

PACS numbers: 44.30.+v, 05.70.Jk, 05.70.Fh

As the liquid-vapor critical point $(\mathrm{CP})$ is approached, many thermodynamic and transport properties of pure fluids exhibit striking behavior [1]. Notable examples include the divergence of the isothermal compressibility, of the isobaric thermal expansion coefficient, as well as the vanishing behavior of the thermal diffusivity. In fluids maintained at constant volume, heat may be transferred by both thermal diffusion and adiabatic compression. This mechanism was coined "the piston effect" because an expanding hot boundary layer acts as a piston to heat the interior of the fluid [2-5]. In pure fluids heat diffusion is slower whereas the piston effect is faster as their CP is approached. The latter process becomes dominant in near-critical fluids, leading to a very fast thermalization. The adiabatic nature of the fast thermal equilibration in supercritical homogeneous fluid samples has been confirmed by numerous experiments performed in weightlessness, where convection is absent, and is now well established [6-9].

The thermal equilibration of near-critical inhomogeneous fluids is more complicated to characterize. The most striking consequence of adiabatic heat transfer in inhomogeneous fluids is the possibility to observe local overheating when a positive temperature quench (initial temperature $T_{i}$, final temperature $T_{f}>T_{i}$ ) is imposed by the thermostat, as previously noted by Onuki and Ferrel for two-phase fluids [5]. More precisely, it is expected that the local temperature can exceed the final temperature. Indeed numerical simulations of the thermal response (without convection) of two-phase near-critical fluids submitted to Earth's gravity $[10,11]$ showed a weak overheating. We have previously observed a large overheating in the gas phase of a two-phase sample during an experiment dedicated to the study of boiling in near-critical fluids [12]. This transient phenomenon would be unthinkable for purely diffusive, isobaric equilibration processes, since it would violate the second law of thermodynamics. But when the volume of the fluid sample is constant, this phenomenon is made possible even at a large distance from the CP. Indeed, the temperature change in the fluid is not only due to heat diffusion but also due to adiabatic compression, i.e., to the work done by pressure changes that are subject to mechanical equilibrium conditions and not to thermal equilibrium conditions.

In order to analyze this paradoxical thermal behavior, we performed a dedicated experiment following the FrenchAmerican GMSF scientific program using the Alice 2 facility onboard the MIR station during the French-Russian Perseus mission (February 1999-August 1999). We used $\mathrm{SF}_{6}$ below its critical point, where the differences between the thermophysical properties of the vapor and the liquid increase according to universal power laws with the critical temperature difference $T_{C}-T$ [1] ( $T$ is the temperature, $T_{C}$ is the liquid-vapor critical temperature). Far from the $\mathrm{CP}$ we took advantage of the large thermophysical differences between the gas and the liquid to magnify the difference between the adiabatic responses of each phase. In this Letter we report on the results of this study of the piston effect in two-phase fluids, whose thermocompressible nature permits the gas to become hotter than the heating wall and the heat to flow from cold to hot.

The Alice 2 facility [13] integrates a management system for diagnostics and stimuli with a fine regulation system that controls the temperature of a sample cell. A Yellow Spring Instruments thermistor (2 s rise time, $100 \mu \mathrm{K}$ accuracy) imbedded in the sample cell unit near the fluid volume was used to follow the temperature evolution of the cell walls (labeled $T_{W}$ ). The sample cell was made of a $\mathrm{CuCoBe}$ alloy and filled with pure $\mathrm{SF}_{6}$ at its critical density $( \pm 0.1 \%)$. The fluid volume was a cylinder (12 $\mathrm{mm}$ internal diameter, $6.7 \mathrm{~mm}$ thickness) sandwiched between two parallel sapphire windows. The inner surface of one of the sapphire windows was coated with a dielectric that formed an interferometric mirror. The image of the sample obtained through the 
Twyman-Green interferometer was recorded by a CCD camera at a frequency of $25 \mathrm{~Hz}$. The initial two-phase distribution in low gravity consisted of a gas bubble surrounded by the liquid that wets the walls of the cell, whose interferometric image is shown in Fig. 1a. The bubble touched the sapphire windows, and its cross section is shown schematically in Fig. 1b. Three Thermometrics B10 thermistors ( $10 \mathrm{~ms}$ rise time, $500 \mu \mathrm{K}$ accuracy, $0.2 \mathrm{~mm}$ diameter) were placed in the fluid volume, allowing local measurements of the fluid temperature. Two of them (labeled Th1 and Th2) were located close to the cell wall (Th1: $0.7 \mathrm{~mm}$, Th2: $1.3 \mathrm{~mm}$ ) and were always observed to be in the liquid. The temperature measured by Th1 (respectively, Th2) is labeled $T_{L}^{1}$ (respectively, $T_{L}^{2}$ ). The third thermistor (Th3) was mounted in the center of the cell, so that in the low gravity environment of the MIR station (residual acceleration of the order of $10^{-4} \mathrm{~g}$ where $\mathrm{g}$ is the acceleration of Earth's gravity) the gas bubble, of volume fraction $\frac{1}{2}$, was always found to contain $\mathrm{Th} 3$ (see Fig. 1a). The temperature measured by Th3 is labeled $T_{G}$. The measuring frequency of $T_{L}^{1}, T_{L}^{2}$, and $T_{G}$ (respectively, $T_{W}$ ) was $25 \mathrm{~Hz}$ (respectively, $1 \mathrm{~Hz}$ ) during the first $5 \mathrm{~min}$ following the quench, then $0.1 \mathrm{~Hz}$ during the next $55 \mathrm{~min}$.

A series of rapid positive temperature quenches of amplitude $\Delta T=T_{f}-T_{i}=100,50,25$, and $20 \mathrm{mK}$ for $T_{i}$ ranging from $T_{C}-10.1 \mathrm{~K}$ to $T_{C}-0.1 \mathrm{~K}$ was performed. A typical evolution of $T_{W}, T_{L}^{1}, T_{L}^{2}$, and $T_{G}$ during a $100 \mathrm{mK}$ quench is shown in Fig. 2. During each quench the temperatures measured in the liquid $T_{L}^{1}$ and $T_{L}^{2}$ always

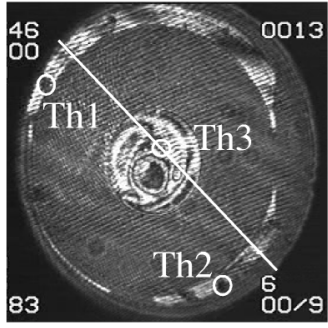

(a)

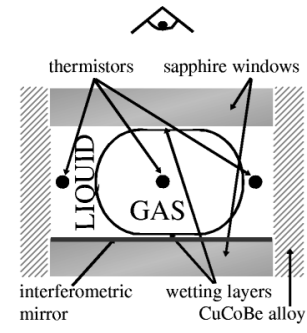

(b)

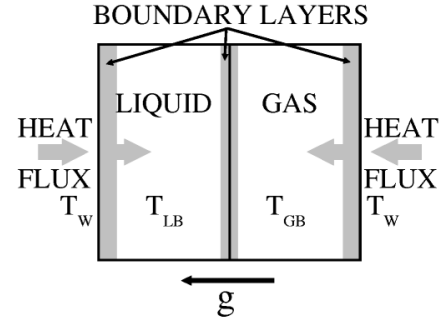

(c)

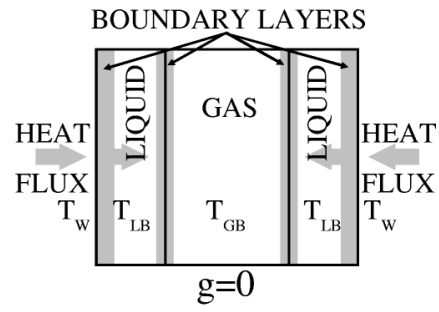

(d)
FIG. 1. (a) Interferometric cell image during a $+100 \mathrm{mK}$ quench $\left(T_{i}=T_{C}-10.1 \mathrm{~K}\right)$. Circles: Th1, Th2, and Th3 thermistor positions. Solid line: thermistor thread. (b) Schematic cell cross section (see text). Sketch of the phase distribution (c) under Earth's gravity and (d) in low gravity. Gray: boundary layers. Arrows show the direction of heat transfer and $\mathrm{HBL}$ expansion. remained smaller than the wall temperature $T_{W}$. Before the end of each quench $T_{G}$ passed well beyond $T_{L}^{1}, T_{L}^{2}$, and more strikingly also well beyond $T_{W}$. In order to compare the different gas overheatings, the temperature rise in the gas $\delta T_{G}$ was reduced by the temperature rise of the cell walls $\delta T_{W}$ (the rise of any temperature $T_{X}$ is defined as $\left.\delta T_{X}=T_{X}-T_{i}\right)$. The behavior of its maximum $\left(\frac{\delta T_{G}}{\delta T_{W}}\right)_{\max }$ as a function of $T_{C}-T_{i}$ is plotted in the inset of Fig. 2. It exhibits an extremum of $123 \%$ around $T_{C}-T_{i} \simeq 5-7 \mathrm{~K}$. The vanishing differences in thermophysical properties between gas and liquid at the CP imply that, asymptotically near the $\mathrm{CP}$, gas and liquid show the same thermal response. On the other hand, far from the $\mathrm{CP}$, the efficiency of the adiabatic heating is reduced and the heat transfer is mainly diffusive, a situation that prevents the gas overheating. The existence of an extremum for the gas overheating can then result from the competition between an increasing efficiency of the adiabatic heating process and a decreasing difference of behavior between gas and liquid as the $\mathrm{CP}$ is approached.

Note that (i) the gas overheating occurred at short time scales and (ii) its amplitude is very large compared to the predictions for the fluid under the Earth's gravity when convection is absent $[10,11]$. Such large overshoots were numerically observed by Eicher [14] in 1D simulations of the thermal response to a heat pulse. This configuration had the gas surrounded by liquid in a spherical cell, in the absence of gravity. Our observations confirm the key role of the actual phase distribution on the thermal relaxation in two-phase near-critical fluids. The strong differences between such thermal responses, with or without gravity, are clearly not due to convection. They can be explained as follows. Since the piston effect is driven by the expansion of the hot boundary layer at the cell wall, its efficiency

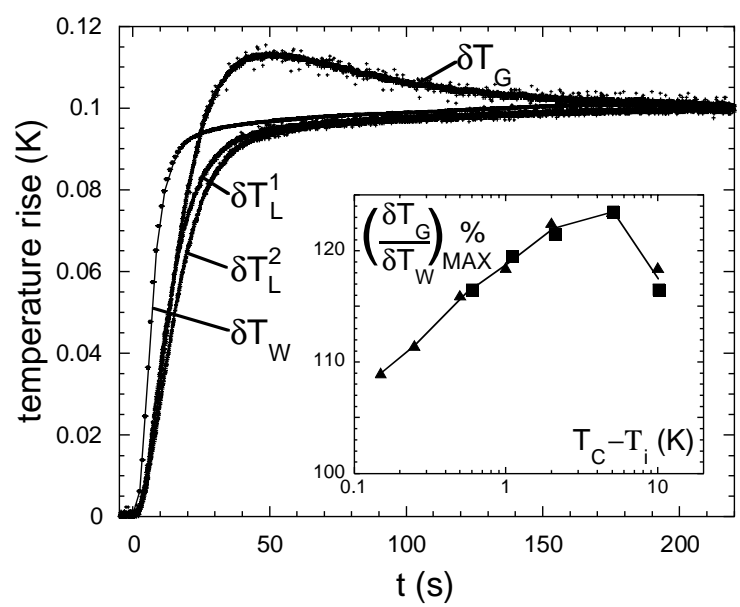

FIG. 2. Relative temperature evolution at the cell wall $\delta T_{W}$, in the liquid $\delta T_{L}^{1}, \delta T_{L}^{2}$, and in the gas $\delta T_{G}\left(\delta T_{X}=T_{X}-T_{i}, X=\right.$ $L, G, W)$ during a temperature quench of $+100 \mathrm{mK}$ from $T_{i}=$ $T_{C}-10.1 \mathrm{~K}$. Inset: $100 \times\left(\frac{\delta T_{G}}{\delta T_{W}}\right)_{\max }$ expressed as a function of $T_{C}-T_{i}$. Quench amplitudes: $+100 \mathrm{mK}$ (squares), $+50 \mathrm{mK}$ (triangles). Solid lines are a guide for the eye. 
depends crucially on whether the thermal boundary layer develops in the liquid phase or in the gas phase. For the typical two-phase distribution under Earth's gravity (liquid at the bottom, gas at the top when we neglect the thin wetting layer surrounding the gas) as sketched in Fig. 1c, two kinds of hot boundary layers (HBL) develop at the cell wall during a quench, one in gas and one in liquid. We define the bulk liquid temperature $T_{L B}$ (respectively, gas $\left.T_{G B}\right)$ as the temperature of the region between the liquid (respectively, gas) HBL at the cell wall and the boundary layer developing at the gas-liquid interface, which is heated only by adiabatic compression. The HBL in the gas (respectively, liquid) stops its expansion when $T_{G B}$ (respectively, $T_{L B}$ ) reaches $T_{W}$. When $T_{G B}$ has reached $T_{W}$, the gas HBL may even contract (cold piston effect) if the liquid HBL continues to expand in order to compensate gas overheating. In this simplified description, the piston effect cannot generate any overheating. We note that the overheatings observed numerically under the Earth's gravity are very weak (of order $1 \%$ of the quench amplitude). Moreover they do not occur in the bulk fluid, but in the slowly diffusing HBLs, where they are the result of the local addition of the diffusive and the adiabatic heat transfer. This scenario is noticeably different from what happens in a gas-liquid system in low gravity, as in our experiment, sketched in Fig. 1d. A video analysis shows that the contact area between the gas bubble and the windows is less than $6 \%$ of the overall heating area. We neglect this area and assume that the HBLs develop in liquid only. Consequently, when the liquid layer that isolates the gas from the cell wall is thick enough, the adiabatic heat transfer stops when $T_{L B}$ has reached $T_{W}$ (Fig. 1d). Before the equilibration of $T_{L B}$ and $T_{W}$, the bulk gas (as defined as the homogeneous interior of the bubble, excluding any boundary layer; see Fig. 1d) is compressed in the same manner as the bulk liquid. Since the gas bubble is not in contact with the heating wall, its temperature has no influence on the temperature gradient at the wall that drives the expansion of the liquid HBL. $T_{G B}$ can thus exhibit a large overshoot, due to the larger thermal response to a pressure change of the gas compared to the liquid. The gas bubble is thus an isolated hot region.

We now compare the temperature evolution in the gas and in the liquid at short times. $\delta T_{W}$ reaches $90 \%$ of $\Delta T$ in less than $10 \mathrm{~s}$, and its evolution is linear in time during at least $5 \mathrm{~s}$ (Fig. 2). The evolution of $\delta T_{L}^{1}, \delta T_{L}^{2}$, and $\delta T_{G}$ are also found to be linear in time during at least $5 \mathrm{~s}$ (Fig. 2). We calculate the corresponding growth rates over $5 \mathrm{~s}$. The quantities $\left(\frac{d\left(\delta T_{G}\right)}{d t} / \frac{d\left(\delta T_{L}^{1}\right)}{d t}\right)-1$ and $\left(\frac{d\left(\delta T_{G}\right)}{d t} / \frac{d\left(\delta T_{L}^{2}\right)}{d t}\right)-1$ are plotted as a function of $T_{C}-T_{i}$ in Fig. 3. It appears that they are equal for $T_{C}-T_{i}<$ $0.6 \mathrm{~K}$. More precisely, $T_{L}^{1}=T_{L}^{2}=T_{L B}$ for $T_{C}-T_{i}<$ $0.6 \mathrm{~K}$, showing that Th1 and Th2 remain out of the HBL and measure the homogeneous temperature of the bulk liquid which undergoes only adiabatic heating. This result

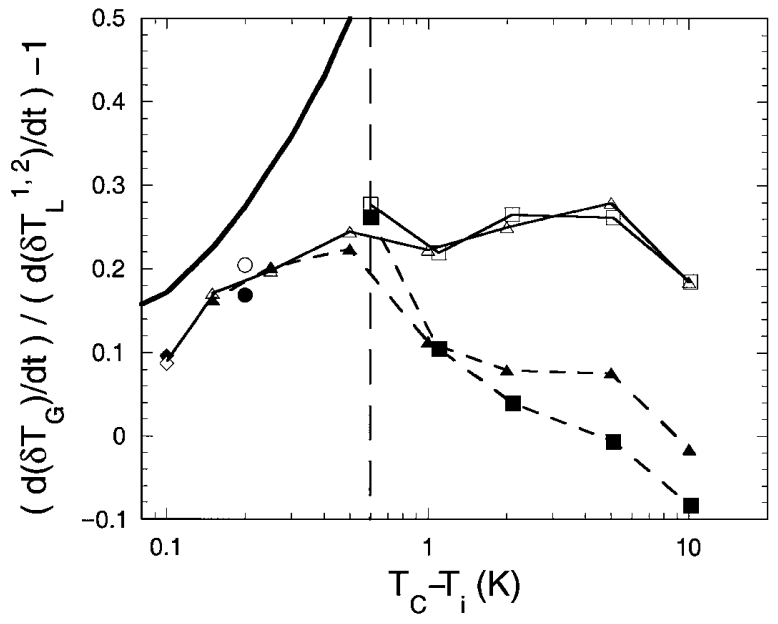

FIG. 3. $\left(\frac{d\left(\delta T_{G}\right)}{d t} / \frac{d\left(\delta T_{L}^{1}\right)}{d t}\right)-1$ (solid symbols, dotted line as a guide for the eye) and $\left(\frac{d\left(\delta T_{G}\right)}{d t} / \frac{d\left(\delta T_{L}^{2}\right)}{d t}\right)-1$ (open symbols, solid line as a guide for the eye) as a function of $T_{C}-T_{i}$. Quench amplitudes: $+100 \mathrm{mK}$ (squares), $+50 \mathrm{mK}$ (triangles), $+25 \mathrm{mK}$ (circles), $+20 \mathrm{mK}$ (diamonds). The vertical dashed line separates the regions where $\mathrm{Th} 1$ and $\mathrm{Th} 2$ are (left) in the bulk liquid $\left(T_{L}^{1}=T_{L}^{2}=T_{L B}\right)$ and (right) in the liquid HBL. Bold solid curve: $\left[\left(\frac{\partial T}{\partial P}\right)_{S}^{G} /\left(\frac{\partial T}{\partial P}\right)_{S}^{L}\right]-1$ computed using the equation of state of Ref. [16].

is related to the fact that during the quench the liquid HBL extends less as the CP is approached, mainly because of the vanishing behavior of the thermal diffusivity $D_{T}$ [15]. Then, for $T_{C}-T_{i} \geq 0.6 \mathrm{~K}$, Th1 and Th2 measure the temperature inside the inhomogeneous liquid HBL.

In the present case of a positive temperature quench on a two-phase equilibrium, the phase density change occurs by diffusive mass transport coupled to diffusive heat transport through the gas-liquid interface, in order to equilibrate the chemical potential [5,11]. For a near-critical two-phase fluid, the adiabatic compression is so fast that when it stops only thin boundary layers of gas and liquid close to the interface have equilibrated their temperature and chemical potential. The rest of the gas and the liquid has not yet reached the coexistence state, and in the bulk each phase behaves as if it were monophasic [6]. Neglecting the heat and mass transfer associated with the phase change, the adiabatic heating should induce temperature differences between the bulk gas and the bulk liquid verifying the prediction of Onuki and Ferrel [5]:

$$
\frac{\delta T_{G B}}{\delta T_{L B}}-1=\frac{d\left(\delta T_{G B}\right) / d t}{d\left(\delta T_{L B}\right) / d t}-1=\frac{(\partial T / \partial P)_{S}^{G}}{(\partial T / \partial P)_{S}^{L}}-1,
$$

where $G$ and $L$ on the right-hand side label the isentropic derivative of $T$ with respect to $P$ in one-phase gas and liquid. In Fig. 3, the bold solid line corresponds to the right-hand term of Eq. (1) computed using the equation of state for $\mathrm{SF}_{6}$ given in Ref. [16]. In the range $T_{C}-T_{i}<0.6 \mathrm{~K}$ for which $T_{L}^{1}$ and $T_{L}^{2}$ coincide with $T_{L B}$, the experimental data are weakly below the prediction of 


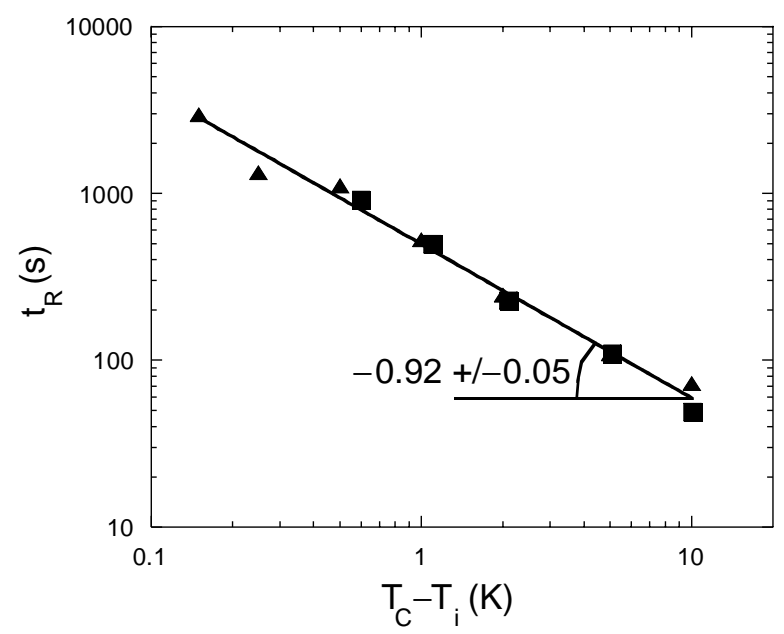

FIG. 4. $t_{R}$ as a function of $T_{C}-T_{i}$ (log-log plot). See Fig. 2 for symbols and Eq. (3) for the fit.

Eq. (1). We attribute this disagreement to heat and mass exchange at the gas-liquid interface so that the compression of the bulk gas and liquid is not perfectly isentropic, as assumed in Eq. (1), and for $T_{C}-T_{i} \geq 0.2 \mathrm{~K}$, this exchange should play an important role, even at short times. Note that a rapid mass exchange at the interface was seen numerically by Zhong et al. [11], but its influence on the temperature evolution was not discussed.

To analyze the long time thermal relaxation of the gas, the decrease of $T_{G}$ towards $T_{f}$ after the maximum overheating is fitted to an exponential law

$$
T_{G}-T_{f}=\theta \exp \left(-\frac{t}{t_{R}}\right),
$$

where the amplitude $\theta$ and the relaxation time $t_{R}$ are the free parameters of the fit. This analytical form is justified by the fact that, at long times, only the (exponential) relaxation of the slowest mode can be observed [17]. The variations of $t_{R}$ as a function of $T_{C}-T_{i}$ (Fig. 4) are fitted to

$$
t_{R}=t_{0}\left(T_{C}-T_{i}\right)^{x},
$$

with $t_{0}$ and $x$ as free parameters of the fit. The best fit value is $x=-0.92 \pm 0.05$ which is close to the opposite value of the effective critical exponent 0.89 of the thermal diffusivity for both gas and liquid [15]. The long time relaxation of the temperature inhomogeneities, initially generated by the adiabatic heat transfer, is diffusive. The thermal isolation of the gas bubble from the cell walls by the liquid prevents a fast relaxation of the large gas overheating by the cold piston effect.

In conclusion, the measurements in low gravity of the temperature evolution of gas and liquid phases near the $\mathrm{CP}$ during positive quenches highlight the key role of the phase distribution on the adiabatic heat transfer. A strong gas overheating, due to the adiabatic heat transfer, is permitted by the isolation of the gas bubble from the heat sources. The strong dependence on the phase localization of the thermal behavior of such two-phase near-critical fluids shows that the real distribution of gas and liquid must be taken into account to understand energy transfer. How heat can be transferred from cold to hot can be understood by a thermomechanical coupling thanks to the piston effect. We finally note that our study is a step towards a full understanding of phase separation and boiling mechanisms in dense compressible fluids.

This work was supported by CNES. The authors thank the team of the Perseus mission and the MIR station crew for their technical support, especially J. F. Zwilling and the French cosmonaut J. P. Haigneré. J. H. gratefully acknowledges the support of NASA.

[1] H. E. Stanley, Introduction to Phase Transitions and Critical Phenomena (Clarendon, Oxford, 1971).

[2] A. Onuki, H. Hao, and R. A. Ferrell, Phys. Rev. A 41, 2256 (1990).

[3] H. Boukari, J. N. Shaumeyer, M.E. Briggs, and R.W. Gammon, Phys. Rev. A 41, 2260 (1990).

[4] B. Zappoli et al., Phys. Rev. A 41, 2264 (1990).

[5] A. Onuki and R. A. Ferrel, Physica (Amsterdam) 164A, 245 (1990).

[6] J. Straub, L. Eicher, and A. Haupt, Phys. Rev. E 51, 5556 (1995).

[7] R. de Bruijn et al., Physica (Amsterdam) 242A, 119 (1997).

[8] R. A. Wilkinson et al., Phys. Rev. E 57, 436 (1998).

[9] Y. Garrabos et al., Phys. Rev. E 57, 5665 (1998).

[10] J. Straub and L. Eicher, Phys. Rev. Lett. 75, 1554 (1995).

[11] F. Zhong and H. Meyer, Phys. Rev. E 53, 5935 (1996).

[12] Y. Garrabos et al., in Proceedings of the CNRS PR2M-1185 Research Group Meeting, Oléron, 1998 [J. Chim. Phys. 96, 1066 (1999)].

[13] R. Marcout et al., Microgravity Q. 5, 162 (1995).

[14] L. Eicher, Ph.D. thesis, Technische Universitaet Munchen, Munchen, 1996.

[15] P. Jany and J. Straub, Int. J. Thermophys. 8, 165 (1987).

[16] A. K. Wyczalkowska and J. V. Sengers, J. Chem. Phys. 111, 1551 (1999).

[17] R. P. Behringer, A. Onuki, and H. Meyer, J. Low Temp. Phys. 81, 71 (1990). 